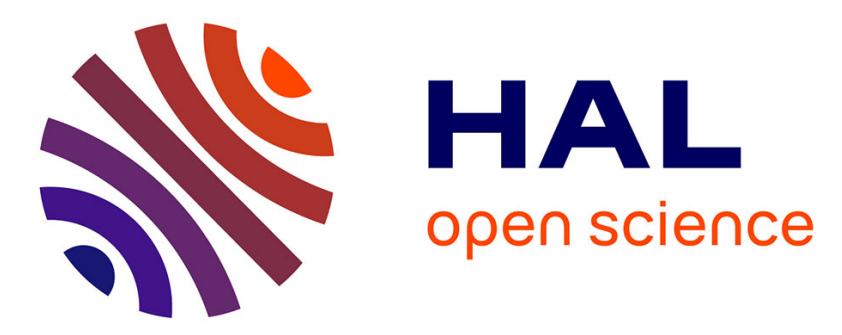

\title{
Singular-perturbations-based analysis of synchronization in heterogeneous networks: A case-study
}

\author{
Mohamed Maghenem, Elena Panteley, Antonio Loria
}

\section{To cite this version:}

Mohamed Maghenem, Elena Panteley, Antonio Loria. Singular-perturbations-based analysis of synchronization in heterogeneous networks: A case-study. 55th IEEE Conference on Decision and Control (CDC 2016), Dec 2016, Las Vegas, NV, United States. pp.2581-2586, 10.1109/CDC.2016.7798651. hal-01357285

\section{HAL Id: hal-01357285 \\ https://hal.science/hal-01357285}

Submitted on 5 Mar 2020

HAL is a multi-disciplinary open access archive for the deposit and dissemination of scientific research documents, whether they are published or not. The documents may come from teaching and research institutions in France or abroad, or from public or private research centers.
L'archive ouverte pluridisciplinaire HAL, est destinée au dépôt et à la diffusion de documents scientifiques de niveau recherche, publiés ou non, émanant des établissements d'enseignement et de recherche français ou étrangers, des laboratoires publics ou privés. 


\title{
Singular-Perturbations-Based Analysis of Synchronization in Heterogeneous Networks: A Case-Study
}

\author{
Mohamed Maghenem Elena Panteley Antonio Loría
}

\begin{abstract}
In recent work, we laid the basis of an analysis framework for the study of heterogeneous networks. In essence, it is postulated that in a heterogeneous network a collective nontrivial behaviour arises, which may be modelled as a dynamical system itself. Then, we say that the networked systems synchronize or, more precisely, achieve dynamic consensus if they adopt this emergent behaviour. In this paper we consider the casestudy of coupled Andronov-Hopf oscillators. We establish that the emergent dynamics, which is of the same nature as a single oscillator, is orbitally stable. Then, we show that the trajectories of the individual oscillators tend to a neighbourhood of the stable orbit. For the first time in the study of synchronization, the analysis is based on singular-perturbations theory; we show that the emergent dynamics corresponds to a slow system while the synchronization errors form a fast dynamics.
\end{abstract}

\section{INTRODUCTION AND MOTIVATION}

Synchronization of networked systems, particularly of oscillators, plays an important role in different research disciplines such as physics, ecology, economics, medicine etc. If all the the nodes in the network are identical and the interconnection gain is sufficiently high, it is expectable that the networked systems synchronize. Furthermore, it is intuitively clear that a synchronized behaviour of such a network corresponds, roughly speaking, to all nodes adopting the motion of a single isolated unit.

In the case of heterogeneous networks, however, the paradigm of asymptotic synchronization is much more complex: the existence of a synchronization manifold is not guaranteed hence, in lieu of adopting the behaviour of one of the nodes, some type of internal model is necessary and sufficient for synchronization [1] and, in general, only "practical" synchronization is usually ensured for heterogeneous networks [2]-[5]. However, in spite of numerous article sin which stability theory is used to analyze heterogeneous networks, -see e.g. [2], [3], [6]-[9], the question of characterizing classes of heterogeneous networks that may achieve asymptotic synchronization remains largely open.

In [5], [10] a novel framework of analysis for heterogeneous networks was developped upon the premise that the collective behavior of network-interconnected systems is dichotomic: it consists, on one hand, in a "weighted averaged" motion determined by the so-called emergent dynamics and, on the other, in the dynamics of the synchronization errors of each individual unit in the network, relatively to the collective behaviour.

M. Maghenem is with Univ Paris-Saclay, A. Loria and E. Panteley are with the CNRS. L2S-CentraleSuplec, 91192 Gif-sur-Yvette, France. E-mail: loria@lss.supelec.fr. E. Panteley is also with ITMO University, Kronverkskiy av. 49, Saint Petersburg, 197101, Russia. This article is supported by Government of Russian Federation (grant 074-U01).
From a dynamical systems viewpoint, the emergent dynamics pertains to an "average" of the units' drifts. This dynamics is intrinsic to the newtork as it is determined by the network's graph, but it is independent of the intensity of the interconnections. Then, if all units behave (asymptotically) as the emergent dynamics, we say that the network achieves dynamic consensus. Thus, dynamic consensus generalizes more usual paradigms in which all units achieve a synchronization manifold or a simple equilibrium point. For instance, in the case of a (homogeneous) network of oscillators it is imaginable that they adopt an averaged oscillatory motion. Yet, in the case of heterogeneous networks the collective behaviour must typically be described via a dynamic system.

Based on the ideas in [5], [10], in this paper we analyze the collective behaviour of a network of heterogeneous Andronov-Hopf oscillators. Assuming that the network is parameterized by the (scalar) coupling gain, and using a linear change of coordinates, we regard the network dynamics as a system that evolves in two different time scales: a slow one in which the emergent dynamics evolves and a fast one, which corresponds to that of the synchronization error dynamics. Remarkably, such model reformulation naturally leads us to recast the analysis into the realm of stability of dynamical systems via singular-perturbations theory [11]-[15]. To the best of our knowledge, this is the first article where such methods are applied to the analysis of synchronization in heterogeneous networks.

The rest of the paper is organized as follows. In Section III we describe the system's model and we recast the synchronization problem in terms of a stability one for singularly-perturbed systems. In Section IV we present our main result. Section V is devoted to some technical proofs. Some concluding remarks are provided in Section VI.

\section{PReliminaries AND NOtATION}

We study the stability of a class of systems on the $n$ dimensional complex plane $\mathbb{C}^{n}$, given by

$$
\dot{z}=f(z), \quad z \in \mathbb{C}^{n}
$$

where $f$ is locally Lipschitz. To that end, we first need to introduce some notations and recall a few concepts and facts.

For a vector $z \in \mathbb{C}^{n}$, let $z^{*}$ denote its transpose conjugate. Then, let the norm operator $|\cdot|$ be defined as $|z|=\sqrt{z^{*} z}$ and, for a closed set $\omega \subset \mathbb{C}^{n}$ we define the usual distance to the set as $|z|_{\omega}:=\inf _{y \in \omega}|z-y|$. We also recall the $\mathcal{L}_{\infty}$ norm,

$$
|f|_{\infty}:=\limsup _{t \rightarrow \infty}|f(t)| \text {. }
$$


If the system (1) admits a periodic solution $\left(t, z_{\circ}\right) \mapsto z^{*}$ starting at $z^{*}\left(0, z_{\circ}\right)=z_{\circ}$, we say that it admits a closed periodic orbit $\gamma \subset \mathbb{C}$ which contains all and only the elements in the image of $\left(t, z_{\circ}\right) \mapsto z^{*}$. For such systems, we study the following stability property $-c f$. [16].

Definition 1 (Orbital Stability): The closed periodic orbit $\gamma$ of the system (1) is said to be orbitally stable if for each $\varepsilon>0$ there exist $\delta>0$ and $T \geq 0$, such that

$$
\forall\left|z_{\circ}\right|_{\gamma} \leq \delta \quad \Longrightarrow \quad\left|z\left(t, z_{\circ}\right)\right|_{\gamma} \leq \varepsilon, \quad \forall t \geq T .
$$

Moreover, the invariant orbit $\gamma$ is said to be asymptotically orbitally stable, if it is orbitally stable and attractive that is, there exists $r>0$ such that

$$
\lim _{t \rightarrow \infty}\left|z\left(t, z_{\circ}\right)\right|_{\gamma}=0, \quad \forall\left|z_{\circ}\right|_{\gamma} \leq r .
$$

For periodic systems,

$$
\dot{x}=A(t) x, \quad A(t+\alpha)=A(t), \quad \forall t \geq t_{0}
$$

orbital stability may be inferred using the so-called characteristic multipliers [17, Section III.7]. After Floquet theory, [18], [19], there exists an $(\alpha)$-periodic matrix $P(t)$ and a constant matrix $B$, such that the fundamental matrix, solution of (3), has the following form:

$$
X(t)=P(t) e^{B t}
$$

and the non-singular periodic transformation $x=p(t) y$ transforms the system (3) into

$$
\dot{y}=B y .
$$

Then, the characteristic multipliers of $A(t)$ are defined as the eigenvalues of the matrix $e^{B \alpha}$. Then, after [17] we have the following.

Lemma 1 (Characterization of Orbital Stability):

Consider the autonomous system $\dot{z}=f(z)$, which admits a closed periodic orbit $\gamma$ and let $\varphi(t)$ be a periodic solution belonging to $\gamma$. Then, the periodic orbit $\gamma$ is (locally) orbitally asymptotically stable if the Jacobian matrix $A(t):=\frac{\partial f}{\partial z}(\varphi(t))$ has a unique characteristic multiplier equal to $1, \mu_{1}=1$ and all the others are inside the unit circle. See [Section VI.2. [17]].

\section{MODEL DESCRIPTION AND PROBLEM FORMULATION}

The unforced Andronov-Hopf equation, which represents a normal form of the bifurcation carrying the same name, is given by

$$
\dot{z}=-\nu|z|^{2} z+\mu z
$$

where $z \in \mathbb{C}$ denotes the state of the oscillator and $\nu, \mu \in \mathbb{C}$ are constant parameters; $\nu:=\nu_{R}+j \nu_{I}$ and $\mu:=\mu_{R}+j \mu_{I}$.

The analysis of (6) is well documented in the literature. For instance, via Lyapunov-exponents-based methods, as in [20] and [19], or using Lyapunov's direct method, as in [21] and [22]. Under these conditions, the behaviour of the Andronov-Hopf oscillator on the phase plane is illustrated in Fig. 1.

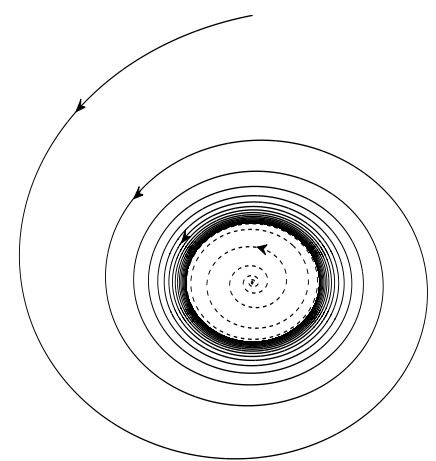

Fig. 1. Trajectories of the Andronov-Hopf oscillator on the complex plane. If $\mu_{R}>0$ the origin is unstable but all trajectories tend to a stable limitcycle of radius $r=\sqrt{\mu_{R} / \nu_{R}}$

For the sequel, we single out some important properties of Andronov-Hopf oscillators and, without loss of generality, we assume that $\nu=1$. First, it is clear that the solutions are globally ultimately bounded. More precisely, for any $\delta>0$, there exists $T>0$ such that

$$
\left|z\left(t, z_{\circ}\right)\right| \leq \sqrt{\mu_{\mathrm{R}}}+\delta \quad \forall t \geq T .
$$

Furthermore, if $\mu_{\mathrm{R}}>0$ then (6) admits an invariant set that is composed of two disjoint invariant subsets, that is,

$$
\mathcal{W}:=\left\{z \in \mathbb{C}:|z|=\sqrt{\mu_{\mathrm{R}}}\right\} \cup\{z=0\} .
$$

Moreover the invariant orbit $\left\{z \in \mathbb{C}:|z|=\sqrt{\mu_{\mathrm{R}}}\right\}$ is almost globally asymptotically stable and the origin $\{z=0\}$ is antistable -see [23]. In addition, for the perturbed AndronovHopf oscillator

$$
\dot{z}=\left[-|z|^{2}+\mu\right] z+u
$$

the set $\mathcal{W}$ is attractive:

Proposition 1: [4] Consider the system (8) with initial conditions $z_{\circ} \in \mathbb{C}$ and let the set $\mathcal{W}$ be defined by (7). Then, the system (8) has the asymptotic gain property, i.e., there exists a class $\mathcal{K}_{\infty}$ function $\eta$ such that

$$
\limsup _{t \rightarrow+\infty}\left|z\left(t, z_{\circ}, u\right)\right|_{\mathcal{W}} \leq \eta\left(|u|_{\infty}\right) .
$$

\section{A. Network model and problem formulation}

Let us consider now a network of $n$ forced normalized Andronov-Hopf oscillators,

$$
\dot{z}_{i}=-\left|z_{i}\right|^{2} z_{i}+\mu_{i} z_{i}+u_{i}
$$

where $i \in[1, \ldots, n], z_{i} \in \mathbb{C}$ is the state of the $i$ th oscillator, the complex parameter $\mu_{i}=\mu_{R i}+i \mu_{I i} \in \mathbb{C}$ defines its amplitude and frequency, and $u_{i}$ is a control input. We assume that the oscillators are interconnected via diffusive coupling, i.e.,

$$
u_{i}=-\gamma\left[l_{i 1}\left(z_{i}-z_{1}\right)+\ldots+l_{i n}\left(z_{i}-z_{n}\right)\right]
$$

where, $l_{i j}$ are real, non-negative numbers denoting the individual interconnections weights and the scalar parameter $\gamma>0$ corresponds to the common coupling strength. Then, let $L$ denote the corresponding Laplacian matrix, and let 
$\boldsymbol{z} \in \mathbb{C}^{n}$ denote the overall network's state, that is $\boldsymbol{z}=$ $\left[z_{1}, \ldots, z_{n}\right]^{\top}$. Replacing (10) in (9) we see that the overall network dynamics takes the form

$$
\dot{z}=f(z)-\gamma L z,
$$

where the function $f: \mathbb{C}^{n} \rightarrow \mathbb{C}^{n}$ is given by

$$
\begin{aligned}
f(\boldsymbol{z}) & :=\left[f_{1}\left(z_{1}\right) \cdots f_{n}\left(z_{n}\right)\right]^{\top}, \\
f_{i}\left(z_{i}\right) & :=-\left|z_{i}\right|^{2} z_{i}+\mu_{i} z_{i} .
\end{aligned}
$$

If the network's graph is connected and undirected, then the corresponding Laplacian matrix $L=L^{\top}$ has exactly one eigenvalue (say, $\lambda_{1}$ ) equal to zero, while others are positive, i.e., $0=\lambda_{1}<\lambda_{2} \leq \ldots \leq \lambda_{n}$. We denote by $v_{1}^{\top}=\frac{1}{\sqrt{n}} \mathbf{1}^{\top}$ the eigenvector associated to the eigenvalue $\lambda_{1}$. Since $L$ is symmetric, its Jordan decomposition can be written as

$$
L=U\left[\begin{array}{ll}
0 & 0 \\
0 & \Lambda
\end{array}\right] U^{\top}
$$

where $\Lambda=\operatorname{diag}\left\{\lambda_{i}(L)\right\}_{i=2, n}>0$ and

$$
U=\left[\begin{array}{ll}
v_{1} & V
\end{array}\right] \text {. }
$$

In turn, $V \in \mathbb{R}^{(n-1) \times n}$ is constituted of the eigenvectors corresponding to the nonzero eigenvalues of $L$, and it satisfies

$$
v_{1}^{\top} V=0, \quad V^{\top} V=I_{n-1} .
$$

Following [5], [10] we propose a change of coordinates that allows to transform the network model in a way to exhibit the emergent dynamics (collective behaviour) and the synchronization error dynamics. Furthermore, we show that the resulting model is in singular-perturbation form, the emergent dynamics corresponding to the slow motion and the synchronization error dynamics being the fast dynamics. To the best of our knowledge, this is the first time that synchronization is studied using singular-perturbations theory.

\section{B. Coordinate transformation and model reformulation}

Following [5], [10] we show that the emergent dynamics is intrinsic to the network. To that end, we use the matrix $U$ which stems from the Jordan decomposition of the Laplacian $L$, to define the new coordinate

$$
\overline{\boldsymbol{z}}:=U^{\top} \boldsymbol{z}
$$

which, in view of (15), we may partition as follows:

$$
\overline{\boldsymbol{z}}=:\left[\begin{array}{c}
z_{m} \\
e_{v}
\end{array}\right]:=\left[\begin{array}{c}
v_{1}^{\top} \boldsymbol{z} \\
V^{\top} \boldsymbol{z}
\end{array}\right]=\left[\begin{array}{c}
\frac{1}{\sqrt{n}} \mathbf{1}^{\top} \boldsymbol{z} \\
V^{\top} \boldsymbol{z}
\end{array}\right] .
$$

Modulo a factor $\sqrt{n}$, the coordinate $z_{m}$ may be regarded as an average of the respective oscillators' states. Such a coordinate is often used in the literature on networked systems to define the synchronization errors as differences between the individual states and the state of the "averaged" unit $-c f$. [2], [3], [9], [10], [24], that is,

$$
e=z-z_{m} v_{1} \text {. }
$$

The second coordinate, $e_{v}$, can be seen as a projection of the synchronization errors (18) onto the subspace that is orthogonal to the vector $v_{1}$. Indeed, note that $e_{v}=V^{\top} e$ since

$$
e_{v}=V^{\top} \boldsymbol{z}=V^{\top}\left(\boldsymbol{z}-z_{m} v_{1}\right)=V^{\top} e .
$$

Moreover, a rather direct computation shows that $e=V e_{v}$.

In the new coordinates, the network equations (11) take the form

$$
\dot{\bar{z}}=U^{\top}[f(\boldsymbol{z})-\gamma L \boldsymbol{z}]
$$

and, using the properties of $L$ and $U$, we obtain

$$
\begin{aligned}
\dot{z}_{m} & =v_{1}^{\top} f\left(V e_{v}+v_{1} z_{m}\right) \\
\dot{e}_{v} & =V^{\top} f\left(V e_{v}+v_{1} z_{m}\right)-\gamma \Lambda e_{v} .
\end{aligned}
$$

Remark 1: It is important to underline that due to the properties of the Laplacian $L$, the $z_{m}$-dynamics, (20), is independent from the the coupling gain $\gamma$.

Now, in the study of synchronization problems of networked systems, it is commonly assumed that the coupling parameter $\gamma$ is large. Therefore, we introduce the singular parameter $0<\varepsilon \ll 1$,

$$
\varepsilon:=\frac{1}{\gamma}
$$

so, dividing by $\gamma$ both sides of (21) the network dynamics equations (20), (21) become

$$
\begin{aligned}
\dot{z}_{m} & =F\left(z_{m}, e_{v}\right) \\
\varepsilon \dot{e}_{v} & =\varepsilon \tilde{G}\left(z_{m}, e_{v}\right)-\Lambda e_{v}=G\left(\varepsilon, e_{v}, z_{m}\right)
\end{aligned}
$$

This system has the standard singular-perturbations form with coordinates $z_{m}$ and $e_{v}$ corresponding to the states of the "slow" and "fast" dynamics of the network respectively. We stress that the model (22), (23) appears naturally, in view of the large coupling parameter $\gamma$ in the network dynamics and the coordinate transformation (16) which reflects the intrinsic properties of the network structure.

\section{Reduced-order model of the network and its properties}

The reduced-order model is obtained from (22), (23) by setting $\varepsilon=0$. Due to the particular structure of the system, notably the fact that $\varepsilon$ appears linearly in the right hand part of (23), the reduced-order model has the simple form

$$
\begin{aligned}
e_{v} & =0 \\
\dot{z}_{m} & =v_{1}^{\top} f\left(v_{1} z_{m}\right),
\end{aligned}
$$

where we used (20) to obtain the reduced dynamics for $z_{m}$.

Using (12) and recalling that $v_{1}=\frac{1}{\sqrt{n}} \mathbf{1}$, we obtain the explicit form of the slow dynamics,

$$
\dot{z}_{m}=-\frac{1}{n}\left|z_{m}\right|^{2} z_{m}+a z_{m}
$$

The parameter $a \in \mathbb{C}$ in this equation corresponds to the average of frequencies and amplitudes of the individual oscillators, i.e.,

$$
a:=a_{R}+i a_{I}:=\frac{1}{\sqrt{n}} \sum_{1}^{n} \mu_{R j}+i \frac{1}{\sqrt{n}} \sum_{1}^{n} \mu_{I j} .
$$


Clearly, the slow reduced-order system (26) corresponds to a single "averaged" Andronov-Hopf oscillator.

Thus, the dynamic model of a network of AndronovHopf oscillators may be regarded as a singularly-perturbed system, in which the slow system (23) has a limit-cycle $\left\{|z|=\sqrt{n a_{R}}\right\}$. The next step in our analysis is to ensure continuity of such behavior for sufficiently small values of the perturbation parameter $\varepsilon$. Based on [12], [15], we prove that the unperturbed limit-cycle $\left\{|z|=\sqrt{n a_{R}}\right\}$ can be continued to a family of limit-cycles with periods that are sufficiently close to the period of the unperturbed one. Then, we use a trajectory-based approach and corresponding tools from dynamical system theory [17], [25], [16] to study the stability of the limit-cycle under sufficiently small perturbations.

\section{MAIN RESUlT}

Our main statement is that there exists a unique periodic orbit for the system (22)-(23) that is locally orbitally asymptotically stable. Then, in view of Lemma 1 this means that all solutions starting away from this orbit tend to it, or to the origin.

Now, in order to state our main results formally, we must introduce a few more definitions and notations. Firstly, we denote by $\gamma_{0}$ a closed orbit of the system (24), (26), that we define as

$$
\gamma_{0}:=\left\{\left(e_{v}, z_{m}\right) \in \mathbb{C}^{n-1} \times \mathbb{C}: e_{v}=0,\left|z_{m}\right|^{2}=n a_{R}\right\} .
$$

Then, we define $\gamma_{0}^{\alpha}$ as a set of trajectories of (24), (26) of period $\alpha$ and starting from $\gamma_{0}$, that is,

$$
\begin{aligned}
\gamma_{0}^{\alpha}:=\left\{\left(e_{v 0}, z_{m 0}\right)(t): \mathbb{R}_{\geq 0}\right. & \rightarrow \mathbb{C}^{n-1} \times \mathbb{C}: \\
e_{v 0}(t)=0, z_{m 0}(t) & \left.=\sqrt{n a_{R}} e^{\left[\frac{2 \pi}{\alpha} t+\phi\right] j}, \phi \in \mathbb{R}\right\}
\end{aligned}
$$

where $j:=\sqrt{-1}$. The difference between the sets $\gamma_{0}$ and $\gamma_{0}^{\alpha}$ must be clear. The former is an invariant set (of points) in the state space of the system (24), (26), while $\gamma_{0}^{\alpha}$ is the set of $(\alpha)$-periodic trajectories of the same system starting from initial states in $\gamma_{0}$. Notice that $\gamma_{0}^{\alpha_{\varepsilon}}$ and $\gamma_{0}^{\alpha_{0}}$ denote sets of trajectories describing the same orbit $\gamma_{0}$ at different frequencies.

Now, due to the singular perturbation $\varepsilon$ in the fast dynamics (23), the system (22)-(23) cannot have the same limitcycle as the reduced-order model (24), (26). Hence, in the sequel, we denote by $\gamma_{\varepsilon}$ a closed orbit of the the perturbed system (22)-(23) and, correspondingly, $\gamma_{\varepsilon}^{\alpha_{\varepsilon}}$ denotes the set of trajectories of the same system, describing the orbit $\gamma_{\varepsilon}$ with period $\alpha_{\epsilon}$.

In addition, for the purpose of analysis, we introduce the sets $T_{\rho}$ and $\Gamma_{\rho}^{\alpha_{\varepsilon}}$ which, roughly, correspond to $\rho$-neighborhoods of $\gamma_{0}$ and $\gamma_{0}^{\alpha_{\varepsilon}}$ respectively. For any $\rho \geq 0$, we define the torus $T_{\rho}$, which contains the orbit $\gamma_{0}$, as

$$
\begin{aligned}
T_{\rho}:=\left\{\left(e_{v}, z_{m}\right) \in \mathbb{C}^{n-1} \times \mathbb{C},\right. \text { such that: } \\
\\
\left.\min _{\left|z_{m 0}\right|=\sqrt{n a_{R}}}\left|z_{m}-z_{m 0}\right|+\left|e_{v}\right| \leq \rho\right\}
\end{aligned}
$$

and we define $\Gamma_{\rho}^{\alpha_{\varepsilon}}$ as a set of all continuous $\left(\alpha_{\varepsilon}\right)$-periodic closed trajectories $\left(e_{v p}(t), z_{m p}(t)\right)$ inside $T_{\rho}$, such that there exists $\left(0, z_{m 0}(t)\right) \in \gamma_{0}^{\alpha_{\varepsilon}}$, such that,

$$
\left|e_{v p}(t)\right|+\left|z_{m p}(t)-z_{m 0}(t)\right| \leq \rho, \quad \forall t \geq 0 .
$$

We are now ready to present our main statement.

Theorem 1: Consider the dynamical system (22)-(23). Let $\gamma_{0}$ be a limit cycle for the reduced-order model (24), (26). Then, there exists $\varepsilon^{*}>0$, such that, for all $\varepsilon \in\left(0, \varepsilon^{*}\right]$, the system (22)-(23) admits a unique non trivial $\left(\alpha_{\varepsilon}\right)$-periodic orbit contained in $\gamma_{\varepsilon}^{\alpha_{\varepsilon}}$, which is asymptotically orbitally stable, and $\varepsilon$-close to $\gamma_{0}^{\alpha_{0}}$. Moreover, the trajectories of (22)(23), either converge to $\gamma_{\varepsilon}^{\alpha_{\varepsilon}}$, or to a small neighborhood of the origin.

Remark 2: The previous statement implies that if $\varepsilon \ll 1$, we can expect the trajectories of (22)-(23) to remain close to those of (24), (26).

The proof of Theorem 1 is constructed in three steps. Firstly, for sufficiently small values of $\varepsilon$, we estabish the existence of an $\left(\alpha_{\varepsilon}\right)$-periodic solution of the system (22)-(23) and we show that the period $\alpha_{\varepsilon}$ converges to the period of the reduced-order model, as $\varepsilon \rightarrow 0$. We denote the corresponding orbit by $\gamma_{\varepsilon}$. We also show the exsitence of sufficiently small constants $\varepsilon^{*}, \rho\left(\varepsilon^{*}\right)>0$, and the corresponding torus $T_{\rho}$ such that, for any $\varepsilon \leq \varepsilon^{*}$, the system (22)-(23) has a unique orbit $\gamma_{\varepsilon} \subset T_{\rho}$. -see Lemma 2 below. The second step relies on Lemma 3 of Section $\mathrm{V}$ to prove asymptotic orbital stability of $\gamma_{\varepsilon}$. Finally, the practical attractivity result established by Lemma 1 allows to conclude that, for a sufficiently small values of $\varepsilon$, the trajectories either converge to the torus $T_{\rho}$, hence to $\gamma_{\varepsilon}^{\alpha_{\varepsilon}}$, or to a small neighborhood of the origin of (22)-(23).

An interesting corollary of the main result is the following.

Corollary 1: Consider the dynamical system given by (22), (23). There exists $\varepsilon^{* *} \in\left(0, \varepsilon^{*}\right]$, with $\varepsilon^{*}$ as introduced in Theorem 1, such that for all $\varepsilon \in\left(0, \varepsilon^{* *}\right]$, there exists a positive constant $d>0$ and a set $B_{d} \subset \mathbb{C}$ of zero Riemannian volume, such that:

1) all solutions of (22)-(23) such that $\left|z_{m}\left(t_{0}\right)\right| \geq \sqrt{n a_{R}}+$ $d$, converge to a non trivial $\left(\alpha_{\varepsilon}\right)$-periodic orbit $\gamma_{\varepsilon}^{\alpha_{\varepsilon}}$;

2) all solutions of (22)-(23) satisfying $\sup _{t \geq t_{1}}\left|e_{v}(t)\right| \leq d$ and $z_{m}\left(t_{1}\right) \in \mathbb{C} \backslash B_{d}$, for some $t_{1} \geq t_{0}$, converge to a non trivial $\left(\alpha_{\varepsilon}\right)$-periodic orbit $\gamma_{\varepsilon}^{\alpha_{\varepsilon}}$.

Proof: In view of Lemma 1, for all $d>0$, there exists $\varepsilon^{* *} \in\left(0, \varepsilon^{*}\right]$ such that, for all $\varepsilon \in\left(0, \varepsilon^{* *}\right]$, we have

$$
\lim _{t \rightarrow \infty}\left|e_{v}(t), z_{m}(t)\right|_{\mathcal{W}^{\prime}} \leq d, \quad \forall \varepsilon \leq \varepsilon^{* *}
$$

with $\mathcal{W}^{\prime}:=\gamma_{0} \cup\{0\}$. Hence, the statement in the first item follows since the trajectories of the orbit $\gamma_{\varepsilon}^{\alpha_{\varepsilon}}$ verify (28). This, the fact that $\left|z_{m}\left(t_{0}\right)\right| \geq \sqrt{n a_{R}}+d$, and continuity of the solutions, imply that the trajectories $\left(e_{v}(t), z_{m}(t)\right)$ cannot reach any sufficiently small neighborhood of the origin without crossing the locally attractive orbit $\gamma_{\varepsilon}$.

The proof of the second item uses [Proposition 1, [26]] which establishes the existence of a positive constant $\Delta>0$ such that, for all $0<d \leq \Delta$, there exists a set $B_{d} \subset \mathbb{C}$ 
of zero Riemannian volume, such that all trajectories of the perturbed system (22) starting outside $B_{d}$ (with $e_{v}(t)$ seen as a perturbation), and verifying $\left|e_{v}(t)\right|_{\infty} \leq d$, converge to a sufficiently small neighborhood of the asymptotically stable equilibria of the unperturbed system (i.e., (22) with $e_{v}=0$ ), which is inside the attractive set of $\gamma_{\varepsilon}^{\alpha_{\varepsilon}}$.

\section{SKETCH OF PROOF OF THE MAIN RESULT}

The proof of Theorem 1 relies on singular-perturbations theory -mainly [27], [12], [15]. We also apply the main result of [12] on the continuation of periodic solutions of singularly perturbed systems. In that regard, it is worth emphasizing that some of the best-known results in the area [28] do not apply in the present context because the slowdynamics does not have an equilibrium.

The following lemma that establishes, for all sufficiently small values of $\rho>0$, the existence of sufficiently small values of $\varepsilon$, such that there exists a unique periodic orbit $\gamma_{\varepsilon} \in T_{\rho}$, of period $\alpha_{\varepsilon}$ and sufficiently close to $\alpha_{0}$, which is solution of the perturbed system (22), (23). Moreover, the lemma establishes that all trajectories of (22) and (23) starting from $\gamma_{\varepsilon}$ belong to $\Gamma_{\rho}^{\alpha_{\varepsilon}}$. See [13], [14].

Lemma 2: Consider the dynamical system given by (22) and (23). There exists $\rho^{*}>0$, such that, for all $\rho \leq \rho^{*}$, there exists $\varepsilon_{2}(\rho)>0$ such that, for all $\varepsilon \leq \varepsilon_{2}(\rho)$, there exists a unique sufficiently smooth periodic orbit $\gamma_{\varepsilon} \in T_{\rho}$ that is solution of (22)-(23), tends to $\gamma_{0}$ as $\varepsilon$ tends to 0 , and has period $\alpha_{\varepsilon}$ tending to $\alpha_{0}$ as $\varepsilon$ tends to 0 . Moreover, all the trajectories of (22) and (23) that start from $\gamma_{\varepsilon}$ belong to $\Gamma_{\rho}^{\alpha_{\varepsilon}}$.

Proof: The first part of the proof relies on a statement from [12] after which the $\left(\alpha_{0}\right)$-periodic orbit $\gamma_{0}$ may be continued to a family of $\left(\alpha_{\varepsilon}\right)$-periodic orbits $\gamma_{\varepsilon}$ when:

1) the Jacobian matrix

$$
\frac{\partial G\left(e_{v}, z_{m 0}, 0\right)}{\partial e_{v}}
$$

does not contain a hyperbolic equilibrium point for all $\left(0, z_{m 0}\right) \in \gamma_{0}$ and

2) the linearization of (26) around any trajectory belonging to $\gamma^{\alpha_{0}}$ has a unique characteristic multiplier equal to 1 .

The first item holds since

$$
\frac{\partial G\left(e_{v}, z_{m 0}, 0\right)}{\partial e_{v}}=-\Lambda \text {. }
$$

To verify the second item we study the local orbital attractivity of $\gamma_{0}$ under the non perturbed dynamics (24) and (25). In view of the non-analyticity of $F\left(e_{v}, z_{m}\right)$ with respect to its arguments, the linearization of (26) around $\left(0, z_{m 0}(t)\right)$ fails in $\mathbb{C}$ (i.e., one cannot find a complex matrix such that $\left.\dot{\tilde{z}}=A\left(z_{m 0}\right) \tilde{z}\right)$. Hence, we rewrite $(26)$ in $\mathbb{R}^{2}$, we get:

$$
\begin{aligned}
& \dot{x}_{m}=-\frac{1}{n}\left(x_{m}^{2}+y_{m}^{2}\right) x_{m}+a_{R} x_{m}-a_{I} y_{m} \\
& \dot{y}_{m}=-\frac{1}{n}\left(x_{m}^{2}+y_{m}^{2}\right) y_{m}+a_{R} y_{m}+a_{I} x_{m} .
\end{aligned}
$$

Now, the linearized form of (29) around

$$
\left[\begin{array}{l}
x_{m 0} \\
y_{m 0}
\end{array}\right]=\left[\begin{array}{l}
\left(n a_{R}\right)^{\frac{1}{2}} \cos \left(a_{I} t+\phi\right) \\
\left(n a_{R}\right)^{\frac{1}{2}} \sin \left(a_{I} t+\phi\right)
\end{array}\right],
$$

for some $\phi \in \mathbb{R}$, is

$$
\left[\begin{array}{l}
\dot{\delta} x_{m} \\
\dot{\delta y_{m}}
\end{array}\right]=\underbrace{\left[\begin{array}{cc}
-2 x_{m 0}^{2} & -2 x_{m 0} y_{m 0}-a_{I} \\
-2 x_{m 0} y_{m 0}+a_{I} & -2 y_{m 0}^{2}
\end{array}\right]}_{A_{1}\left(z_{m 0}(t)\right)}\left[\begin{array}{l}
\delta x_{m} \\
\delta y_{m}
\end{array}\right] .
$$

We establish local orbital attractivity of $\gamma_{0}^{\alpha_{0}}$ using the following non-singular periodic transformation:

$$
y=P^{-}(t) x
$$

where,

$$
P^{-}(t)=\left[\begin{array}{cc}
\sin \left(a_{I} t+\phi\right) & -\cos \left(a_{I} t+\phi\right) \\
\cos \left(a_{I} t+\phi\right) & \sin \left(a_{I} t+\phi\right)
\end{array}\right]
$$

and $P^{-}=P^{\top}$. Differentiating on both sides of (30) we obtain

$$
\dot{y}=B y=\left[\begin{array}{cc}
0 & 0 \\
0 & -2 a_{R}
\end{array}\right] y
$$

and we conclude the exponential stability for the radial component of the error coordinates. Which implies the local orbital attractivity of the circular orbit $\gamma_{0}^{\varepsilon}$. This establishes the second item above, since the characteristic multipliers of $A_{1}\left(z_{m 0}(t)\right)$ are 1 and $e^{-2 a_{R} \alpha_{0}}$.

After [12], we conclude that there exists $\rho^{*}>0$ such that, for all $\rho \leq \rho^{*}$, there exists $\varepsilon_{1}(\rho)>0$ such that, for all $\varepsilon \leq \varepsilon_{1}(\rho)$, there exists a unique sufficiently smooth $\left(\alpha_{\varepsilon}\right)$-periodic orbit $\gamma_{\varepsilon} \in T_{\rho}$, solution of (22) and (23), which tends to $\gamma_{0}$ as $\varepsilon$ tends to 0 , and the corresponding period $\alpha_{\varepsilon}$ tends to $\alpha_{0}$ as $\varepsilon$ tends to 0 .

To prove the second part of the lemma, we use Tikhonov's theorem -see e.g., Theorem 11.1. [27]. Consider the $\left(\alpha_{\varepsilon}\right)$-periodic trajectory $\left(e_{v p}(t), z_{m p}(t)\right) \in \gamma_{\varepsilon}$ solution of the perturbed system (22)-(23) describing the periodic orbit, and the $\left(\alpha_{0}\right)$-periodic trajectory $\left(e_{v 0}(t), z_{m 0}(t)\right) \in \gamma_{0}^{\alpha_{0}}$ solution of the unperturbed system (24), (26), such that:

$$
\left|e_{v p}(0)-e_{v 0}(0)\right|+\left|z_{m p}(0)-z_{m 0}(0)\right| \leq O(\varepsilon) .
$$

This choice is possible due to the $(\varepsilon)$-closeness of $\gamma_{\varepsilon}$ to $\gamma_{0}$. Then using Theorem 11.1. [27], we conclude that for all $t \in\left[0, \alpha_{\varepsilon}\right]:$

$$
\left|e_{v p}(t)-e_{v 0}(t)\right|+\left|z_{m p}(t)-z_{m 0}(t)\right| \leq O(\varepsilon) .
$$

Then, using the $(\varepsilon)$-closeness of $\alpha_{\varepsilon}$ to $\alpha_{0}$, we conclude that there exists $\left(e_{v 1}(t), z_{m 1}(t)\right) \in \gamma_{0}^{\alpha_{\varepsilon}}$ such that

$$
\left|e_{v 1}(0)-e_{v 0}(0)\right|+\left|z_{m 1}(0)-z_{m 0}(0)\right| \leq O(\varepsilon)
$$

and, for all $t \in\left[0, \alpha_{\varepsilon}\right]$,

$$
\left|e_{v 1}(t)-e_{v 0}(t)\right|+\left|z_{m 1}(t)-z_{m 0}(t)\right| \leq O(\varepsilon)
$$

The latter implies that, for all $t \in\left[0, \alpha_{\varepsilon}\right]$,

$$
\left|e_{v p}(t)-e_{v 1}(t)\right|+\left|z_{m p}(t)-z_{m 1}(t)\right| \leq O(\varepsilon) .
$$


Moreover, since $\left(e_{v 1}(t), z_{m 1}(t)\right)$ and $\left(e_{v p}(t), z_{m p}(t)\right)$ have the same period $\alpha_{\varepsilon}$, we conclude that for all $t \geq 0$ :

$$
\left|e_{v p}(t)-e_{v 1}(t)\right|+\left|z_{m p}(t)-z_{m 1}(t)\right| \leq O(\varepsilon) .
$$

it thus follow that there exists $\varepsilon_{3}(\rho)>0$ such that $O\left(\varepsilon_{3}\right) \leq$ $\rho$, imply that taking $\varepsilon_{2}(\rho)=\min \left\{\varepsilon_{2}(\rho), \varepsilon_{3}(\rho)\right\}$, we get $\left(e_{v p}(t), z_{m p}(t)\right) \in \Gamma_{\rho}^{\alpha_{\varepsilon}}$.

The following lemma establishes that, for all sufficiently small values of $\rho>0$, there exist sufficiently small values of $\varepsilon>0$, such that one can establish local asymptotic orbital stability of all periodic orbits inside $\Gamma_{\rho}^{\alpha_{\varepsilon}}$ and solution (22) and (23), with an estimation of the attractive set. The proof is omitted due to space constraints.

Lemma 3: Consider the dynamical system given by (22), (23). Let $\bar{\varepsilon}>0$ be such that for all $\varepsilon \leq \bar{\varepsilon}$, the system admits a periodic orbit $\gamma_{\varepsilon}$ and the corresponding torus $T_{\rho}$. Then, there exists $\varepsilon^{* *} \leq \bar{\varepsilon}$ and $\rho^{* *}=\rho^{* *}\left(\varepsilon^{* *}\right)>0$ such that for all $\varepsilon \leq \varepsilon^{* *}$ the corresponding oribit $\gamma_{\varepsilon}$ of system (22), (23) is asymptotically orbitally stable with domain of attraction $\mathcal{D}_{\varepsilon} \subset \mathbb{C}^{n}$ which contains $T_{\rho^{* *}}$, that is, $T_{\rho^{* *}} \subset \mathcal{D}_{\varepsilon}$ for all $\varepsilon \leq \varepsilon^{* *}$.

Proof of theorem 1: Let $\rho \leq \min \left\{\rho^{*}, \rho^{* *}\right\}$ and $\varepsilon \leq \varepsilon^{* *}$, be such that $T_{\rho}$ is inside the domain of attraction of each $\gamma_{\varepsilon}^{\alpha_{\varepsilon}}$, solution of (22) and (23). After Lemma 3, such a choice of $\rho$ is possible because the domain of attraction of each $\gamma_{\varepsilon}^{\alpha} \subset T_{\rho}$ is independent of $\varepsilon$ and $\rho$ when $\rho \leq \rho^{* *}$, and $\varepsilon \leq \varepsilon^{* *}$. Then, taking $\varepsilon \leq \min \left\{\varepsilon^{* *}, \varepsilon_{2}(\rho)\right\}$, after Lemma 2, there exists a unique $(\alpha)$-periodic orbit $\gamma_{\varepsilon}^{\alpha} \in T_{\rho}$ that is an orbitally exponentially stable solution of (22) and (23), such that $T_{\rho}$ is contained in its domain of attraction.

Finally, taking $\varepsilon^{*} \leq \min \left\{\varepsilon^{* *}, \varepsilon_{2}(\rho), \varepsilon_{1}(\rho)\right\}$, using Lemma 1, we conclude either Attractivity of $T_{\rho}$, at this case the trajectory will converge to $\gamma_{\varepsilon}^{\alpha_{\varepsilon}}$, otherwise it converges to a $\rho$-neighborhood of 0 , which conclude the proof of the theorem.

\section{CONCLUSION}

For a case-study, we formulated and solved a problem of asymptotic synchronization of heterogeneous systems as a problem of orbital stability. For the first time in the literature, the analysis is carried out using singular-perturbations theory. Further research is being carried out to generalize these methods beyond the example of Andronov-Hopf oscillators, along the lines of [10].

\section{REFERENCES}

[1] P. Wieland and F. Allgöwer, "An internal model principle for consensus in heterogeneous linear multi-agent systems," in Estimation and Control of Networked Systems, pp. 7-12, 2009.

[2] J. M. Montenbruck, M. Bürger, and F. Allgöwer, "Practical synchronization with diffusive couplings," Automatica, vol. 53, pp. 235-243, 2015.

[3] T. Liu, D. Hill, and J. Zhao, "Output synchronization of dynamical networks with incrementally-dissipative nodes and switching topology," IEEE Trans. on Circ. Syst. I: Fundamental Theory and Applications, vol. 62, no. 9, pp. 2312-2323, 2015.
[4] E. Panteley, A. Loría, and A. E. Ati, "On the analysis and control design for networked stuart-landau oscillators with applications to neuronal populations," in Proc. 54th. IEEE Conf. Decision Contr., 2015.

[5] E. Panteley, "A stability-theory perspective to synchronisation of heterogeneous networks." Habilitation à diriger des recherches (DrSc dissertation). Université Paris Sud, Orsay, France, 2015.

[6] A. Y. Pogromski and H. Nijmeijer, "Cooperative oscillatory behavior of mutually coupled dynamical systems," IEEE Transactions on Circuits and Systems I: Fundamental Theory and Applications, vol. 48, no. 2, pp. 152-162, 2001.

[7] L. Zhu, Z. Chen, and R. H. Middleton, "A general framework for robust output synchronization of heterogeneous nonlinear networked systems," IEEE Transactions on Automatic Control, vol. 61, no. 8, pp. 2092-2107, 2016. DOI: 10.1109/TAC.2015.2492141.

[8] P. DeLellis, M. Di Bernardo, and D. Liuzza, "Convergence and synchronization in heterogeneous networks of smooth and piecewise smooth systems," Automatica, vol. 56, pp. 1-11, 2015.

[9] E. Panteley, A. Loria, and L. Conteville, "On practical synchronization of heterogeneous networks of nonlinear systems," in Proc. IEEE American Control Conference, (Chicago, IL, USA), pp. 5359-5364, 2015.

[10] E. Panteley and A. Loría, "Synchronization and dynamic consensus of heterogeneous networked systems," IEEE Trans. Automat. Control, 2016. Conditionally accepted.

[11] A. N. Tikhonov, "Systems of differential equations containing small parameters in the derivatives," Matematicheskii sbornik, vol. 73, no. 3 , pp. 575-586, 1952.

[12] D. V. Anosov, "Limit cycles of systems of differential equations with small parameters in the highest derivatives," Matematicheskii Sbornik, vol. 92, no. 3, pp. 299-334, 1960.

[13] J. K. Hale and G. Seifert, "Bounded and almost periodic solutions of singularly perturbed equations," J. Math. Analys. and. Appl., vol. 3, no. 1 , pp. $18-24,1961$.

[14] K. W. Chang, "Almost periodic solutions of singularly perturbed systems of differential equations," J. Diff. Eqs., vol. 4, no. 2, pp. 300307, 1968.

[15] N. Fenichel, "Geometric singular perturbation theory for ordinary differential equations," J. Diff. Eqs., vol. 31, no. 1, pp. 53-98, 1979.

[16] M. Urabe, Nonlinear autonomous oscillations, vol. 34 of Mathematics in science and engineering. Academic Press, 1967.

[17] J. K. Hale, Ordinary differential equations, vol. 21 of Pure and applied mathematics. Krieger Publishing Company, 1969.

[18] G. Floquet, "Sur les quations diffŕentielles lináires à coefficients périodiques," Annales de l'École Normale Supérieure, no. 12, pp. 47$88,1883$.

[19] L. Perko, Differential Equations and Dynamical Systems. Springer, 2000.

[20] Y. A. Kuznetsov, Elements of Applied Bifurcation Theory. Springer, Applied Mathematical Sciences, Vol. 112, 1998.

[21] P. C. Matthews and S. H. Strogatz, "Phase diagram for the collective behavior of limit-cycle oscillators," Phys. Rev. Lett., vol. 65, pp. 17011704, Oct 1990.

[22] Q. C. Pham and J. J. Slotine, "Stable concurrent synchronization in dynamic system networks," Neural Networks, vol. 20, no. 1, pp. 62 77, 2007.

[23] E. Panteley, A. Loría, and A. E. Ati, "On the stability and robustness of stuart-landau oscillators," IFAC-PapersOnLine, vol. 48, no. 11, pp. 645-650, 2015.

[24] P. DeLellis, M. D. Bernardo, and G. Russo, "On quad, lipschitz, and contracting vector fields for consensus and synchronization of networks," Circuits and Systems I: IEEE Transactions on, vol. 58, no. 3, pp. 576-583, 2011.

[25] J. K. Hale, Oscillations in nonlinear systems. Dover publications, 1963.

[26] D. Angeli and L. Praly, "Stability robustness in the presence of exponentially unstable isolated equilibria," IEEE Trans. on Automat. Contr., vol. 56, no. 7, pp. 1582-1592, 2011.

[27] H. K. Khalil, Nonlinear systems. Prentice hall New Jersey, third edition ed., 2002.

[28] P. Kokotović, H. Khalil, and J. O'Reilly, Singular Perturbation Methods in Control: Analysis and Design. Society for Industrial and Applied Mathematics, 1999. 